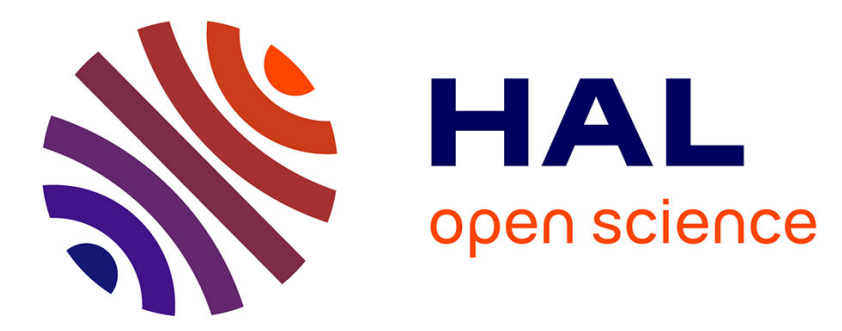

\title{
Avalanche precursors in a frictional model
}

Axelle Amon, Baptiste Blanc, Jean-Christophe Géminard

\section{To cite this version:}

Axelle Amon, Baptiste Blanc, Jean-Christophe Géminard. Avalanche precursors in a frictional model. Physical Review E , 2017, 96 (3), pp.033004. 10.1103/physreve.96.033004 . hal-01512586v2

\section{HAL Id: hal-01512586 https://hal.science/hal-01512586v2}

Submitted on 13 Sep 2017

HAL is a multi-disciplinary open access archive for the deposit and dissemination of scientific research documents, whether they are published or not. The documents may come from teaching and research institutions in France or abroad, or from public or private research centers.
L'archive ouverte pluridisciplinaire HAL, est destinée au dépôt et à la diffusion de documents scientifiques de niveau recherche, publiés ou non, émanant des établissements d'enseignement et de recherche français ou étrangers, des laboratoires publics ou privés. 


\title{
Avalanche precursors in a frictional model
}

\author{
Axelle Amon* \\ Institut de Physique de Rennes, UMR UR1-CNRS 6251, \\ Université de Rennes 1, Campus de Beaulieu, F-35042 RENNES cedex, France
}

Baptiste Blanc ${ }^{\dagger}$ and Jean-Christophe Géminard

Université de Lyon, Laboratoire de Physique, Ecole Normale Supérieure, CNRS, 46 Allée d'Italie, F-69364 Lyon cedex 07, France

(Dated: Received: date / Revised version: date)

\begin{abstract}
We present a one-dimensional numerical model based on elastically coupled sliders on a frictional incline of variable tilt. This very simple approach makes possible to study the precursors to the avalanche and to provide a rationalization of different features that have been observed in experiments. We provide a statistical description of the model leading to master equations describing the state of the system as a function of the angle of inclination. Our central results are the reproduction of large-scale regular events preceding the avalanche, on the one hand, and an analytical approach providing an internal threshold for the outbreak of rearrangements before the avalanche in the system, on the other hand.

PACS numbers: 45.70.Ht,45.70.-n,46.55.+d,45.05.+x
\end{abstract}

\section{INTRODUCTION}

Identifying precursors to avalanches has inspired numerous works with the hope of being able to detect catastrophic events before they occur. A physicist approach consists in simplifying the system in order to single out the fundamental mechanisms underlying the phenomenon. For example, models based on cellular automaton have been proposed to study avalanches [1]. From an experimental point of view, a model set-up to study the behavior of a granular material before an avalanche takes place consists in the progressive inclination of a box filled with a granular material [2-6]. In such experiments, it has been evidenced that the response of the system consists in the superposition of two different behaviors: on the one hand, small rearrangements implying only a few number of grains occuring without any obvious synchronisation across the system and, on the other hand, large correlated events implying a large fraction of the system. Those last events have been shown to emerge from an angle at about half the avalanche angle [4] and then to occur at regular angle increments as the inclination increases until the destabilization of the pile [24]. To our knowledge, no explanation of the regularity of those pre-avalanche events exists in the litterature, nor any numerical observation of the phenomenon [7-9]. Yet, such micro-envents have been observed in different loading configurations $[2,10,11]$ and are reminiscent of precursors observed in studies of the onset of frictional sliding [12].

Here, we present a one-dimensional frictional model that reproduces most of the features reported in the experiments. In particular, the regular micro-ruptures are

\footnotetext{
* axelle.amon@univ-rennes1.fr

$\dagger$ present address:
}

reproduced and an interpretation of the origin of the regularity of the phenomenon as a stick-slip response is given. Synchronization of this periodic response in large, disordered, systems is exhibited by incorporating a global coupling in the model. The physical origin of this coupling is discussed. The simplicity of our model makes possible to provide master equations to describe the evolution state of the system as the inclination is increased. This analytical approach reveals an internal angle signing an intensification of the process.

The article is structured as follow: part II is devoted to the description of the numerical model. Part III presents the results obtained for three typical sets of parameters: a small system without global coupling, a large system without global coupling and finally a large system with global coupling. Part IV details a statistical approach to rationalize our observation. The last part discusses the understanding of the pre-avalanche behavior that can be obtained from our approach and compare our results to previous studies from the litterature.

\section{NUMERICAL MODEL}

\section{A. Description}

The system under study is very close in spirit to the one-dimensional uniform Burridge-Knopoff model [13]. This seminal work has inspired numerous studies aiming at understanding the dynamics of earthquake faults using spring-blocks models [13-15]. Similar models are also studied to understand the onset of frictional sliding between two interfaces in tribological studies $[16,17]$. In our case we build on a model which has been previously proposed to study the effects of minute temperature changes on the stability of granular materials $[18,19]$. The main features of the present study compared to previous ones 
are the following: (i) we consider an homogeneous gravitational loading due to the progressive inclination of the blocks; (ii) we are interested in the evolution of the system from an initial preparation to the critical state which immediately preceed the avalanche; (iii) we propose an implementation of some long-range coupling in the system. We discuss the physical origin of this global coupling in section $\mathrm{V}$.

We consider $N$ identical frictional sliders of mass $m$ lying on a rigid incline making the angle $\alpha$ with the horizontal (Fig. 1). The sliders are connected to one another by linear springs of stiffness $k$. In addition, each slider is connected to a rigid upper plate by a cantilever spring of stiffness $k_{c}$. The upper plate insures a global coupling between the sliders. The springs as well as the upper plate are massless. In this configuration, the sliders are subjected to the elastic forces due to the springs, to their own weight $m g$, with $g$ the acceleration due to gravity, and to the reaction force from the incline which includes a frictional force. The notations used in the following are shown in Fig. 1.

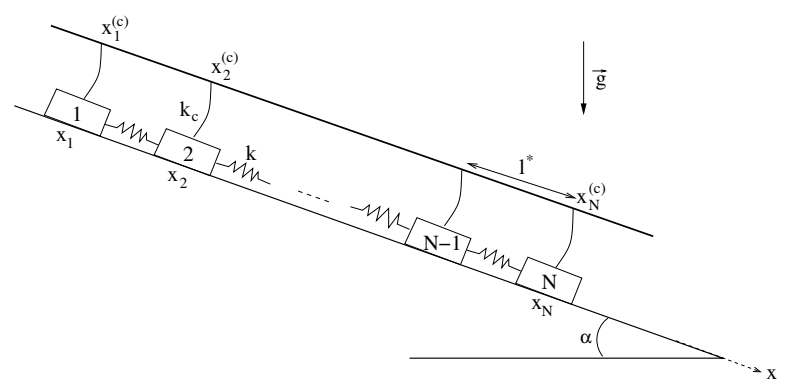

FIG. 1. Sketch of the system under study.

One of the fundamental differences between the system under study and the usual Burridge-Knopoff (BK) models is found in the loading process. Indeed, we focus on the response of the system to a progressive increase of the inclination angle $\alpha$, whereas the system is usually loaded by pulling it at one extremity. We prepare the system at horizontal, and then progressively increase the tilt in a quasi-static way until all the sliders descend the slope, which corresponds to the final avalanche. We are interested in the temporal and spatial distribution of the rearrangements that lead to the avalanche. In particular, we want to study how the distribution of the static frictional forces exerted on the sliders are modified from an initially uniform preparation because of the progressive loading.

Another difference is found in the modeling of the frictional contact. Whereas, in most of the BK-models velocity weakening of the friction force is introduced, we here characterize the frictional contact between the incline and the sliders by static and dynamic frictional coefficients. However, we consider that, due to the heterogeneity of the local properties of the incline surface, the static coefficient $\mu_{s, n}$ takes a different value for each of the individual sliders, indexed by $n$ (from 1 to $N$,
Fig. 1). By constrast, because the sliders in motion see average properties of the incline surface, we consider a single value $\mu_{d}$ of the dynamical frictional coefficient for all the sliders. Note that, in addition, we consider that $\mu_{d}<\mu_{s, n}(\forall n)$. Thus, in summary, we write that the slider $n$ starts moving if

$$
\begin{array}{r}
\left|f_{n+1 \rightarrow n}+f_{n-1 \rightarrow n}+f_{C \rightarrow n}+m g \sin \alpha\right| \\
>\mu_{s, n} m g \cos \alpha
\end{array}
$$

where $f_{n+1 \rightarrow n}$ and $f_{n-1 \rightarrow n}$ are the elastic forces due to the neighbor sliders $n-1$ and $n+1$, and $f_{C \rightarrow n}$ the elastic force due to the cantilever spring that connects it to the upper plate. When the slider $n$ is in motion with the velocity $\dot{x}_{n}$, the frictional force exerted by the incline is

$$
f_{d, n}=-\mu_{d} m g S\left(\dot{x}_{n}\right) \cos \alpha
$$

where $S$ denotes the sign function $[S(u)=1$ if $u>0$ and $S(u)=-1$ if $u<0]$.

\section{B. Dimensionless set of equations}

The dynamics of the system is characterized by the timescale $\tau_{d y n}=\sqrt{m / k}$. We can then introduce the dimensionless time $\tilde{t}=t / \tau_{d y n}$ and position $\tilde{x}=x / g \tau_{d y n}^{2}$. We then denote $\tilde{l}$ the dimensionless natural length of the springs that link the sliders to one another and define $\xi \equiv k_{c} / k$, the ratio of the stiffnesses of the two kinds of springs introduced previously.

Using these latter dimensionless variables, we can write the equations governing the dynamics of a slider in motion. We have (Eq. 2):

$$
\begin{aligned}
\ddot{\tilde{x}}_{1}= & -\left[\tilde{x}_{1}-\tilde{x}_{2}+\tilde{l}\right]-\xi\left[\tilde{x}_{1}-\tilde{x}_{1}^{(c)}\right] \\
& -\mu_{d} S\left(\dot{\tilde{x}}_{1}\right) \cos \alpha+\sin \alpha \quad(n=1) \\
\ddot{\tilde{x}}_{n}= & -\left[2 \tilde{x}_{n}-\left(\tilde{x}_{n+1}+\tilde{x}_{n-1}\right)\right]-\xi\left[\tilde{x}_{n}-\tilde{x}_{n}^{(c)}\right] \\
& -\mu_{d} S\left(\dot{\tilde{x}}_{n}\right) \cos \alpha+\sin \alpha \quad(n \neq 1, N) \\
\ddot{\tilde{x}}_{N}= & -\left[\tilde{x}_{N}-\tilde{x}_{N-1}-\tilde{l}\right]-\xi\left[\tilde{x}_{N}-\tilde{x}_{N}^{(c)}\right] \\
& -\mu_{d} S\left(\dot{\tilde{x}}_{N}\right) \cos \alpha+\sin \alpha \quad(n=N)
\end{aligned}
$$

where

$$
\tilde{x}_{n}^{(c)}=\frac{1}{N} \sum_{i=1}^{N} \tilde{x}_{i}+\left(n-\frac{N+1}{2}\right) \tilde{l}^{*}
$$

with $\tilde{l}^{*}$ the dimensionless, constant, distance $\left(\tilde{l}^{*} \equiv\right.$ $\left.l^{*} / g \tau_{d y n}^{2}\right)$ between the attachment positions $x_{n}^{(c)}$ of the cantilever springs to the upper plate (Fig. 1, refer to next section addressing the preparation of the system). Eq. (6) reflects the fact that the upper plate moves with the barycenter of the sliders.

Depending on its position indexed by $n$, a slider, initially at rest, starts moving if the following condition is 
fulfilled (Eq. 1):

$$
\begin{aligned}
& \left|\tilde{x}_{2}-\tilde{x}_{1}-\tilde{l}-\xi\left[\tilde{x}_{1}-\tilde{x}_{1}^{(c)}\right]+\sin \alpha\right|>\mu_{s, 1} \cos \alpha \\
& (n=1) \\
& \left|\tilde{x}_{n+1}+\tilde{x}_{n-1}-2 \tilde{x}_{n}-\xi\left[\tilde{x}_{n}-\tilde{x}_{n}^{(c)}\right]+\sin \alpha\right|>\mu_{s, n} \cos \alpha \\
& (n \neq 1, N) \\
& \left|\tilde{x}_{N-1}-\tilde{x}_{N}+\tilde{l}-\xi\left[\tilde{x}_{N}-\tilde{x}_{N}^{(c)}\right]+\sin \alpha\right|>\mu_{s, N} \cos \alpha \\
& (n=N)
\end{aligned}
$$

\section{Numerical method}

In this section, we detail the numerical methods, starting with the crucial preparation of the initial state of the system.

\section{Preparation of the system}

First, the initial set of static frictional coefficients $\mu_{s, n}^{(0)}$ accounting for the contact betweent the slider $n$ and the incline (angle $\alpha=0$, initially) are drawn at random from a Gaussian distribution:

$$
p\left(\mu_{s}\right)=\frac{1}{\sqrt{2 \pi \sigma_{\mu}^{2}}} \exp \left[-\frac{\left(\mu_{s}-\bar{\mu}_{s}\right)^{2}}{2 \sigma_{\mu}^{2}}\right]
$$

where $\bar{\mu}_{s}$ is the mean value and $\sigma_{\mu}$ the width of the distribution. However, if a random value $\mu_{d}$ exceeds $\mu_{s}$, we draw it again at random until we have $\mu_{d}<\mu_{s}$.

Once the initial set of static frictional coefficients $\mu_{s, n}^{(0)}$ is chosen, we set the initial positions of the sliders, $\tilde{x}_{n}^{(0)}$, insuring the mechanical stability of the initial configuration in absence of global coupling (The cantilever springs are deconnected, i.e. $\xi=0$ ). To do so, a set of random tangential forces are drawn according to a uniform distribution in $]-\bar{\mu}_{s}, \bar{\mu}_{s}\left[\right.$. The corresponding positions, $\tilde{x}_{n}^{(0)}$, of the sliders are then computed. When considering the behavior of the system in absence of coupling $(\xi=0)$, the obtained configuration is our initial condition. When the behavior of the system with coupling $(\xi \neq 0)$ is considered, we alter the configuration in the following way. First, we set $\tilde{l}^{*} \equiv\left(\tilde{x}_{N}^{(0)}-\tilde{x}_{1}^{(0)}\right) /(N-1)$, the average distance between the sliders. Then, the coupling is introduced by connecting the cantilever springs to the upper plate (by setting $\xi$ to a non-zero value). In response, some sliders can loose stability. If so, the dynamical equations (3) to (5) are integrated until a mechanically-stable configuration is reached. The final positions, $\tilde{x}_{n}^{(0)}$, of the sliders are then our initial condition.

\section{Numerical integration}

From the initial horizontal state $(\alpha=0)$, the tilt angle $\alpha$ is progressively increased. From the knowledge of the static frictional coefficients $\mu_{s, n}^{(0)}$ and positions $x_{n}^{(0)}$, we can determine the value of the angle $\alpha^{(1)}$ leading to a first rearrangement using Eqs. (7) to (9). The dynamical equations are then solved to obtain the set of new steady positions $x_{n}^{(1)}$ of the sliders that moved. To do so, we set the angle $\alpha$ to $\alpha^{(1)}$ and integrate, using a standard velocity Verlet integrator (with a timestep $\Delta t \ll \tau_{d y n}$ ), the dynamical equations (3) to (5) for all the sliders that enter in motion. At each integration step, we check if any of the motionless sliders is destabilized by any displacement of its neighbors. In case of such event, the integration step is reduced until the initiation of the motion of the corresponding slider. In the same way, we check if any slider in motion comes to rest and we adapt the integration step accordingly. A new value of the static frictional coefficient is drawn at random, according to Eq. (10), for those sliders that come to rest. We consider that the rearrangement ends when all the sliders have come back to rest. The new state of the system is then accounted for by the sets of new steady positions $x_{n}^{(1)}$ and static frictional coefficients $\mu_{s, n}^{(1)}$.

From those new initial conditions the procedure can be iterated, leading to a set of destabilization angles $\alpha^{(k)}$ associated to positions $x_{n}^{(k)}$ and static frictional coefficients

$\mu_{s, n}^{(k)}$. The procedure ends when the angle $\alpha$ reaches a critical value, $\alpha_{c}$, such that all the sliders enter in motion and continuously accelerate downwards. This final angle is, by definition, the avalanche angle.

\section{NUMERICAL RESULTS}

In order to get insights in the behavior of the model, we report numerical results obtained for three typical systems, small and large systems with or without coupling:
A. $-N=10$ sliders without coupling $(\xi=0)$.
B. $-N=400$ sliders without coupling $(\xi=0)$.
C. $-N=400$ sliders with coupling $(\xi \neq 0)$.

In all the simulations we set the parameters to typical values, i.e. $l=10, \mu_{d}=0.55, \bar{\mu}_{s}=0.6$, and $\sigma_{\mu}=0.01$.

\section{A. Small system, no global coupling}

In Fig. 2 we report results obtained for a small system of $N=10$ sliders in absence of coupling $(\xi=0)$.

We observe in Fig. 2a that, far from being uniformly distributed neither between the sliders nor during the inclination process, the displacements are gathered in bursts of displacements of adjacent sliders at given angles separated by quiescent inclination intervals. Moreover, 
the interval between the inclinations angles $\alpha$ that lead to rearrangments seems to be almost constant.

(a)

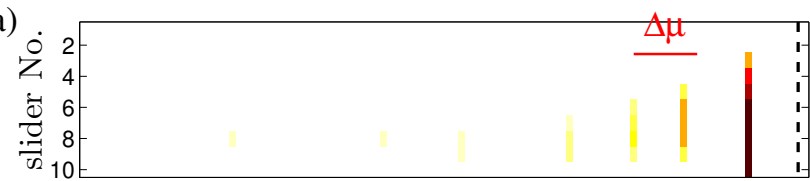

(b)

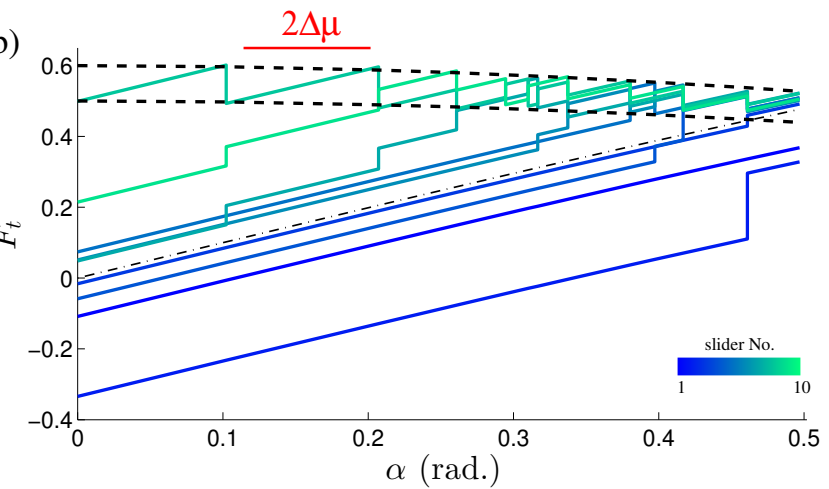

FIG. 2. Behavior of a small system without global coupling upon inclination - (a) Hot map of the displacements $\Delta \tilde{x}_{i}$ vs. angle $\alpha$. Each horizontal line corresponds to one slider. The darker the points are, the larger is the displacement of the slider. The vertical dashed line corresponds to the avalanche angle and $\Delta \mu=\bar{\mu}_{s}-\mu_{d}$. (b) Tangential force $\tilde{F}_{t}\left(\tilde{F}_{t}=\right.$ $\tilde{f}_{n+1 \rightarrow n}+\tilde{f}_{n-1 \rightarrow n}+\tilde{f}_{C \rightarrow n}+\sin \alpha$ ) vs. angle $\alpha$ (The slider number is indicated by the color bar). The upper dashed line is the stability limit: $\bar{\mu}_{s} \cos \alpha$. The lower dashed line is the stability limit $\left(2 \mu_{d}-\bar{\mu}_{s}\right) \cos \alpha$. The dash-dotted line: $\sin \alpha$ $\left[N=10, \xi=0, l=10, \mu_{d}=0.55, \bar{\mu}_{s}=0.6\right.$, and $\left.\sigma_{\mu}=0.01\right]$.

These features can be understood by considering the evolution of the tangential component of the force, due to the springs and the weight, on each of the sliders as function of $\alpha$ (Fig. 2b): $\tilde{F}_{t}=\tilde{f}_{n+1 \rightarrow n}+\tilde{f}_{n-1 \rightarrow n}+\tilde{f}_{C \rightarrow n}+\sin \alpha$. While $\alpha$ is increased, the component of the weight along the incline increases as $\sin \alpha$ (dash-dotted line). Starting from the horizontal $(\alpha=0)$, we observe that the first rearrangment occurs for $\alpha \simeq 0.1$, when the most unstable slider looses stability. The displacement of this slider leads to a drop of the force it is submitted to. Simultaneously, the tangential forces exerted on its two direct neighbors present a sudden increase. At about $\alpha \simeq 0.2$, the same block again looses stability, and drags downwards one of its neighbors which then also looses stability and moves. A further increase of the inclination by a few degrees leads to another rearrangment that involves three sliders, thus a larger region. Sliders, that were unperturbed up-to-now, are now adjacent to sliders that move, and the tangential forces they are subjected to exhibit sudden increases, which places them closer to the instability threshold. Hence, step by step, the bursts of reorganizations imply more and more sliders. Finally, a critical angle, $\alpha_{c} \simeq 0.5$, is reached at which all the sliders destabilize and accelerate down the incline. Note in Fig. $2 \mathrm{~b}$ that the tangential forces exerted on the sliders that rearranged at least once are bounded by two well- defined values

\section{B. Large system, no global coupling}

For a large system, we observe the same typical behavior of the system with the main difference that bursts of rearrangments are observed at different places in the system (Fig. 3a). The picture is more complex as the rearrangments that occur in different regions are not synchronized. In addition, the intervals between the inclinations angles $\alpha$ are, on average, smaller than previously observed in a smaller system. Note also that, upon increasing inclination, the typical size of the active regions increases, which leads to their coalescence.
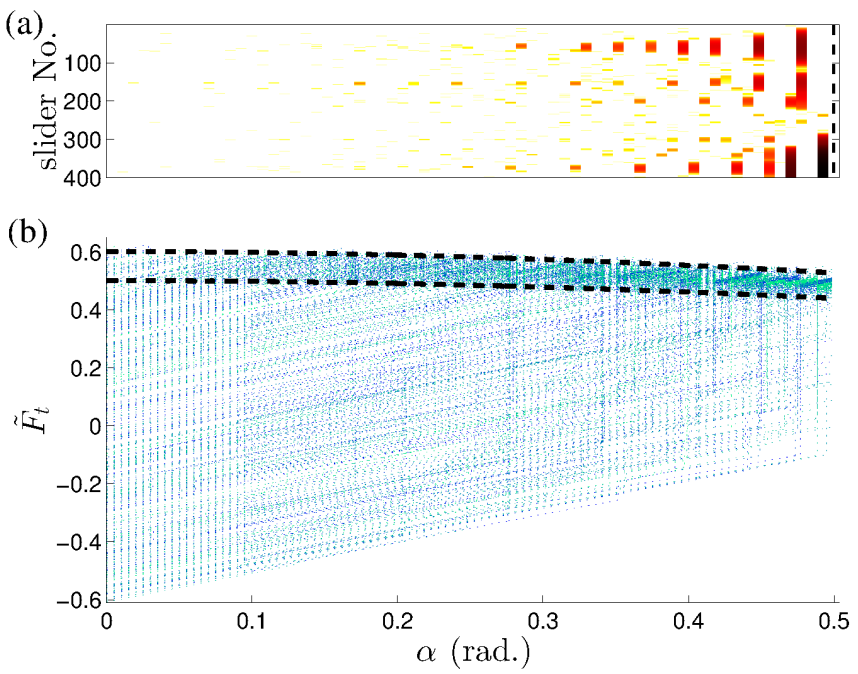

FIG. 3. Behavior of a large system without global coupling upon inclination - (a) Hot map of the displacements $\Delta \tilde{x}_{i}$ vs. angle $\alpha$. (b) Tangential force $\tilde{F}_{t}$ vs. angle $\alpha$ (The slider number is indicated by the color bar). The upper dashed line is the stability limit: $\bar{\mu}_{s} \cos \alpha$. The lower dashed line is the arrest limit $\left(2 \mu_{d}-\bar{\mu}_{s}\right) \cos \alpha[N=400, \xi=0, l=10$, $\mu_{d}=0.55, \bar{\mu}_{s}=0.6$, and $\left.\sigma_{\mu}=0.01\right]$.

Reporting the tangential components of the forces (Fig. 3b), we observe that rearrangements initiate at different locations as the instability threshold is reached by several sliders early in the inclination process. Upon further increase of the inclination angle, each of the active regions evolves, and grows in size, as previously described for the smaller system, which leads, finally, to the avalanche of the whole system.

\section{Large system with global coupling}

From now on, it is of particular interest to observe the behavior of the exact same system when a slight global coupling is introduced. To do so, we set the ratio of the spring constants $k$ and $k_{c}$ to the small, but non zero, value $\xi=0.001$. 
Comparing the behaviour of the system with (Fig. 4) and without (Fig. 3) coupling, we immediately notice the coupling leads to larger rearrangments, involving more sliders and larger displacements than in the uncoupled system. In addition, in Fig. 4, we observe two main active regions involving the two extremities of the system. From an angle of about $\alpha \sim 0.4$, the upper region extends over more than half the size of the system. We also note also that the two active regions synchronize $\alpha \gtrsim 0.42$ even if they do not overlap until the avalanche of the whole system which occurs for $\alpha \simeq 0.51$.
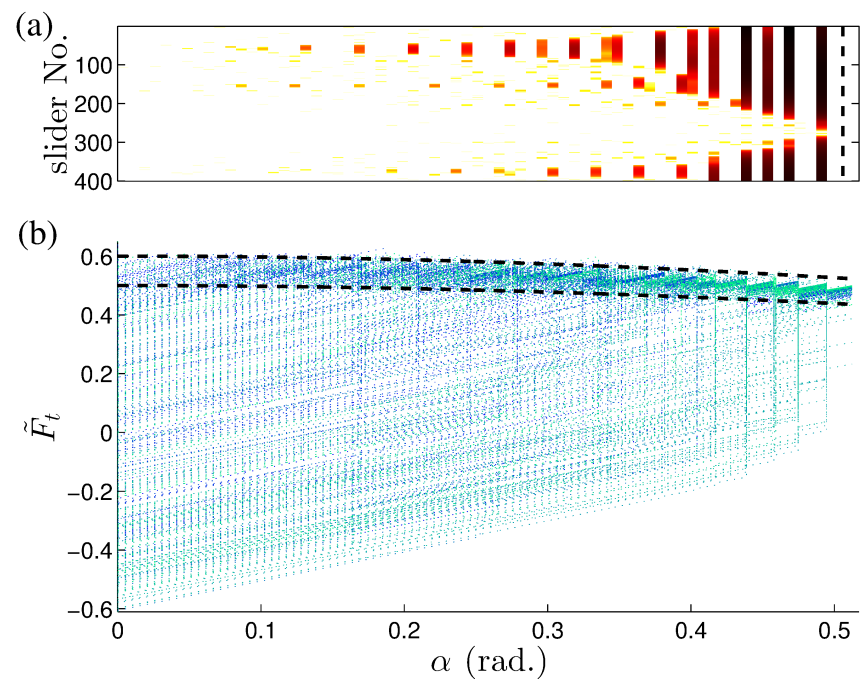

FIG. 4. Behavior of a large system with global coupling upon inclination - (a) Hot map of the displacements $\Delta \tilde{x}_{i}$ vs. angle $\alpha$. (b) Tangential force $\tilde{F}_{t}$ vs. angle $\alpha$ (The slider number is indicated by the color bar). The upper dashed line is the stability limit: $\bar{\mu}_{s} \cos \alpha$. The lower dashed line is the arrest limit $\left(2 \mu_{d}-\bar{\mu}_{s}\right) \cos \alpha\left[N=10, \xi=0.001, l=10, \mu_{d}=0.55\right.$, $\bar{\mu}_{s}=0.6$, and $\left.\sigma_{\mu}=0.01\right]$.

In Fig. 4b, we observe that the tangential components of the forces behave as previously observed in absence of coupling (Fig. 3b). Those components, for the sliders that moved at least once, are bounded by two welldefined values. The effect of the global coupling is clearly visible in the evolution of the tangential component of the forces for $\alpha \gtrsim 0.42$ : for each rearrangment, the tangential component corresponding to the sliders that are not involved exhibit a large increase. This synchronization is also visible by focussing on the behavior of the forces between the two dashed lines in Fig. 3b which correspond to the sliders that moved.

\section{ANALYTICAL MODEL}

We present in this section a simple approach which allows to understand the main features that have been observed in the simulation. First we will study the case of a single slider surrounded by two immobile blocks

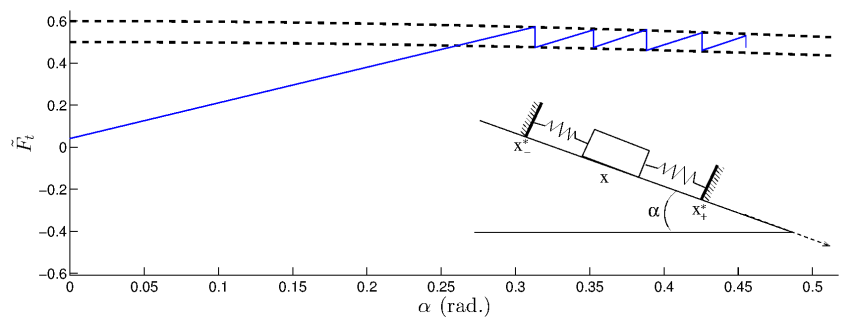

FIG. 5. Tangential force $\tilde{F}_{t}$ vs. angle $\alpha$ - The upper dashed line is the stability limit: $\bar{\mu}_{s} \cos \alpha$. The lower dashed line is the arrest limit $\left(2 \mu_{d}-\bar{\mu}_{s}\right) \cos \alpha$. Inset: sketch of the system under study $\left[\mu_{d}=0.55\right.$ and $\left.\mu_{d}=0.6\right]$.

(Sec. IV A). In Sec. IV B, we will see how a statistical model can be built from the results obtained with a single slider. In this section no global coupling is ever considered $(\xi=0)$. The effect of the coupling, as well as the comparison with experiments, will be the subject of the discussion (Sec. V).

\section{A. A single slider}

Let us first consider a single slider bonded by two springs to two immobile walls as sketched in Fig. 5.

We consider that the walls are located at $x_{-}^{*}$ and $x_{+}^{*}$. Initially, the system is horizontal $(\alpha=0)$ and the slider at stable position $x^{(0)}$. For the sake of simplicity, we assume that static frictional coefficient is constant and equal to $\mu_{s}$. Indeed, in practice $\sigma_{\mu} \ll 2 \mu_{s}$, such that the associated width of the distribution of the static frictional force can be neglected when compared to the variation of the tangential force associated to the choice of $x^{(0)}$.

From Eq. (1), we can determine the angle $\alpha^{(1)}$ corresponding to the limit of stability of the slider:

$$
\begin{aligned}
& \tilde{F}_{t}^{(0)}+\sin \alpha^{(1)}=\mu_{s} \cos \alpha^{(1)}, \\
& \quad \text { where } \tilde{F}_{t}^{(0)}=\tilde{x}_{+}^{*}+\tilde{x}_{-}^{*}-2 \tilde{x}^{(0)}
\end{aligned}
$$

Once $\alpha^{(1)}$ is reached by tilting the system, for this simple case the dynamical Eqs. (2) can be solved analytically, which leads to:

$$
\Delta \tilde{x}^{(1)} \equiv \tilde{x}^{(1)}-\tilde{x}^{(0)}=\left(\mu_{s}-\mu_{d}\right) \cos \alpha^{(1)}
$$

The total tangential force exerted on the block in its new position is

$$
\begin{aligned}
& \tilde{F}_{t}^{(1)}=\mu_{s} \cos \alpha^{(1)}+\Delta \tilde{F}^{(1)}, \\
& \quad \text { where } \Delta \tilde{F}^{(1)}=-2\left(\mu_{s}-\mu_{d}\right) \cos \alpha^{(1)}
\end{aligned}
$$

In the same way, upon further inclination, the slider destabilizes for $\alpha^{(2)}$ verifying:

$$
\left(\tilde{F}_{t}^{(1)}-\sin \alpha^{(1)}\right)+\sin \alpha^{(2)}=\mu_{s} \cos \alpha^{(2)}
$$


Assuming that $\Delta \alpha^{(2)}=\alpha^{(2)}-\alpha^{(1)}$ is small, we get to the first order:

$$
\Delta \alpha^{(2)}=\frac{2\left(\mu_{s}-\mu_{d}\right)}{1+\mu_{s} \tan \alpha^{(1)}}
$$

which remains small provided that $\mu_{s}-\mu_{d} \ll 1$. The reasoning can be reproduced for any of the rearrangments, leading to:

$$
\Delta \alpha^{(i+1)}=\frac{2\left(\mu_{s}-\mu_{d}\right)}{1+\mu_{s} \tan \alpha^{(i)}}
$$

Thus, a generic behavior emerges as shown in Fig. 5 . We observe, first, a loading phase, which depends on the initial position $\tilde{x}^{(0)}$. The tangential component of the force $\tilde{F}_{t}$ increases continuously until it reaches the instability threshold for $\alpha=\alpha^{(1)}$ and suddenly drops by $\Delta \tilde{F}_{t}^{(1)}$. Then, rearrangements occur for successive values $\alpha^{(i)}$ of the inclination angle. They are marked by sudden drops $\Delta \tilde{F}_{t}^{(i)}$ of the tangential component, followed by continuous increase towards the next threshold. In a first order approximation in $\alpha$, the force drops $\Delta \tilde{F}^{(i)}$ do not depend on the initial position of the slider. In addition, for small enough angle $\alpha^{(i)}$, the interval $\Delta \alpha^{(i+1)}$ is almost constant and equal to $\Delta \alpha \simeq 2\left(\mu_{s}-\mu_{d}\right)$. Such periodic behaviour is reminiscent of the well-known stickslip motion. Finally, note that a sliding event leading to a drop $\Delta \tilde{F}_{t}^{(i)}$ of the force exerted to the slider, results in an increase by $\Delta \tilde{F}_{t}^{(i)} / 2=\left(\mu_{s}-\mu_{d}\right) \cos \alpha$ of the force exerted on each of the walls.

\section{B. Statistical point of view}

As will be seen in the discussion (Sec. V), the simple analysis of a single slider between two walls helps to understand most of the features observed for a small system. For large systems however, where the pattern that emerges is more complicated (Sec. III B), a statistical approach is more appropriate to model the system.

In the present section, we seek for master equations describing the evolution of the distribution of the tangential forces $\tilde{F}_{t}$ in a system as a function of the inclination angle $\alpha$. We will make strong approximations to be able to provide an analytical model, but we will compare the solutions we obtain to numerical simulations of large systems in absence of global coupling $(\xi=0)$.

\section{Qualitative analysis}

We report in Fig. 6 the probability distribution function of the tangential forces, $\tilde{F}_{t}$, exerted on the sliders at different values of the inclination angle $\alpha$, in absence of global coupling $(\xi=0)$.

Initially, at $\alpha=0$ (dark blue curve in Fig. 6), the distribution is flat in the interval $]-\mu_{s}, \mu_{s}[$, which corresponds to the initial, random, preparation of the system at horizontal (Sec. II C).

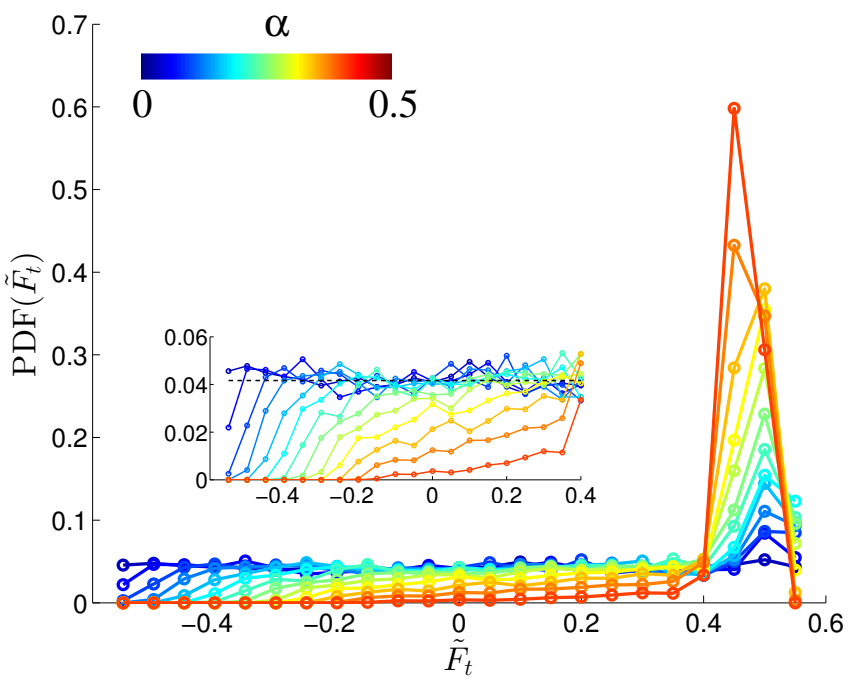

FIG. 6. Probability distribution function of the tangential force $\tilde{F}_{t}$ upon inclination - at different values of the inclination angle - Each distribution corresponds to an average, at a given $\alpha$ (indicated by the colorscale), over 20 numerical runs. Inset: Enlargment of the distribution for $\tilde{F}_{t}<0.4[N=100, \xi=0$, $\tilde{l}=10, \mu_{d}=0.55, \bar{\mu}_{s}=0.6$, and $\left.\sigma_{\mu}=0.01\right]$.

Upon inclination, we observe that the distribution functions exhibits two main parts:

- A rather flat part for $\tilde{F}_{t} \lesssim 0.4$ with a well-defined plateau at the constant initial value. The lowest values of $\tilde{F}_{t}$ are slowly depleted.

- A peak, for $0.4 \lesssim \tilde{F}_{t} \lesssim \bar{\mu}_{s}$, that grows rapidly.

The qualitative behaviour of the distribution function can be understood as follows. Upon inclination, the lowest values of $\tilde{F}_{t}$ are depleted because of the increase of the projection of the weight along the incline. By this simple effect, the lower bound of the distribution increases according to $-\bar{\mu}_{s} \cos \alpha+\sin \alpha$. Conversely, upon increasing inclination, sliders loose stability. The upper bound, which equals $\bar{\mu}_{s} \cos \alpha$ according to (Eq. 1), slightly decreases, but this variation can be neglected at the first order. The value of $\tilde{F}_{t}$ for the sliders that moved remains close to the upper bound. The peak grows as their number increases. This picture holds as long as the plateau and the peak are well separated, i.e. for $\alpha \lesssim 0.25$. Then, the contribution of the sliders that are involved in the rearrangements become predominent.

In order to get more insights in the contribution of the rearrangments, we focus on the distribution of the values of $\tilde{F}_{t}$ for only the sliders that moved (Figure 7). Defining $\tilde{F}_{t}^{*} \equiv\left(2 \mu_{d}-\bar{\mu}_{s}\right) \cos \alpha$, we observe that, for small inclination, the distribution function is peaked around $\tilde{F}_{t} / \tilde{F}_{t}^{*}=1$. This observation shows that the rearrangements initially involve single sliders, the width of the peak is directly linked to the width of the distribution of $\mu_{s}$. By contrast, for large inclination, the distribution 
function exhibits a peak close to the value $\tilde{F}_{t} / \tilde{F}_{t}^{*} \simeq 1.1$, which is an upper limit for all the distribution functions.

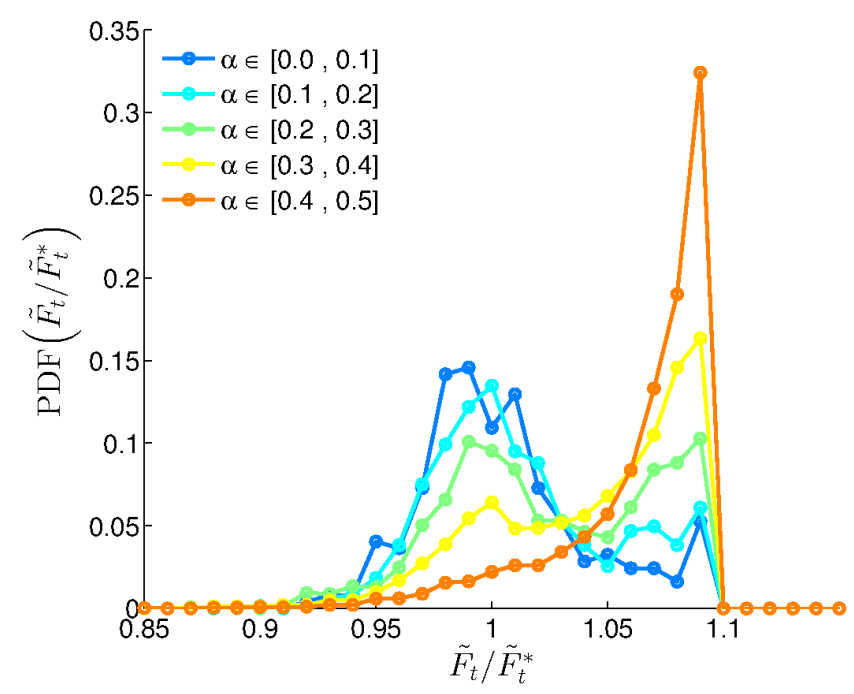

FIG. 7. Probability distribution function of the ratio $\tilde{F}_{t} / \tilde{F}_{t}^{*}$ for the sliders that moved upon inclination - The data are from the 20 same numerical runs as in Fig. $6[N=100, \xi=0$, $\tilde{l}=10, \mu_{d}=0.55, \bar{\mu}_{s}=0.6$, and $\left.\sigma_{\mu}=0.01\right]$.

We can understand the accumulation of the tangential forces at this limit value in the following way. First, let us mention that $\tilde{F}_{t} / \tilde{F}_{t}^{*} \simeq 1.1$ corresponds to $\tilde{F}_{t}=\mu_{d} \cos \alpha$. For rearrangements implying several interacting sliders, the movement becomes complex enough for the vanishing velocities not to be determined by a sinusoidal movement, but by the overall slowing down of the dynamics due to dissipation. At the limit of vanishing accelerations, the sum of elastic forces and weight on each block compensates the dynamical friction so that, when the block stops, the value of the resultant tangential force is typically less than $\mu_{d} \cos \alpha$, thus controlled by the dynamical frictional coefficient.

In conclusion, after a sliding event, for the sliders implied in the event, the tangential forces $\tilde{F}_{t}$ drop by a quantity comprised between $\Delta \mu \cos \alpha$ and $2 \Delta \mu \cos \alpha$ with $\Delta \mu=\bar{\mu}_{s}-\mu_{d}$. This is the main ingredient of the model we present in the next section IV B 2.

\section{Model}

Here we present a model based on the prior observations. We consider in Fig. 8 a schematic representation as a bar histogram of the distribution function reported in Fig. 6. To do so, we discretize the probability density function in bins of width $2 \Delta \mu$ with $\Delta \mu=\bar{\mu}_{s}-\mu_{d}$. In addition, in a first approach, we neglect the effect of the inclination on the upper bound, which we assume to equal $\bar{\mu}_{s}$. We denote $\nu$ the number of bins of width $2 \Delta \mu$, starting from the upper bound, $\bar{\mu}_{s}$, and including the lower bound, $\sin \alpha-\bar{\mu}_{s}$. Thus, $\nu$ is a function of $\alpha$ that decreases with $\alpha$ as: $\nu(\alpha)=\left\lceil\frac{2 \bar{\mu}_{s}-\sin \alpha}{2 \Delta \mu}\right\rceil$. For convenience, the bins are numbered from large to small forces such that the bin No. 1 denotes the peak on the righthand-side, corresponding to the peak of the distribution. We denote $P_{i}$ the height of bin No. i. With these definitions, the probability for a slider to be submitted to a tangential force belonging to the bin No. $\mathrm{i}$ is given by $2 \Delta \mu P_{i}$.

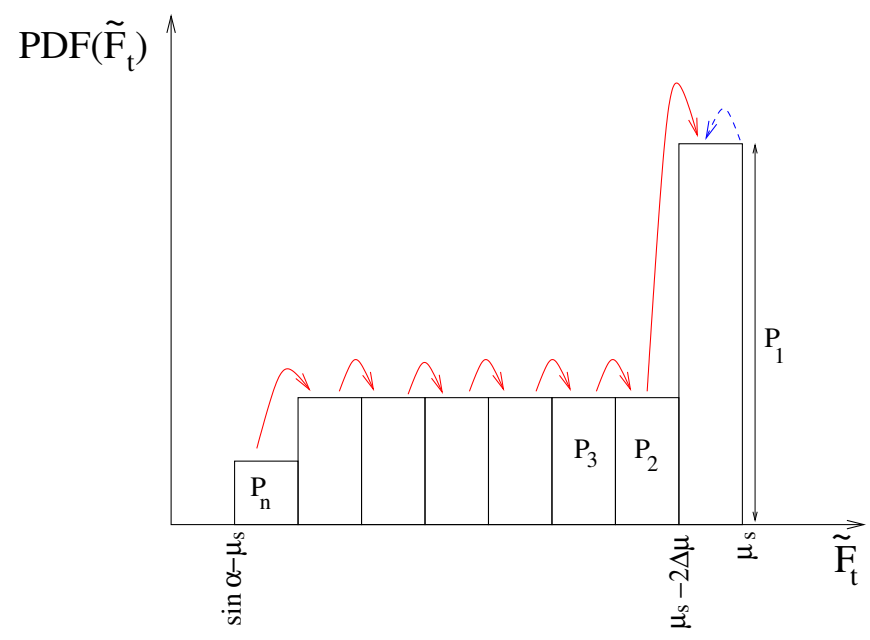

FIG. 8. Schematic representation of the histogram equivalent to the probability distribution function of the tangential force, $\tilde{F}_{t}$ at a given inclination angle $\alpha$. The arrows indicate the main modification of the distribution upon further inclination by $\delta \alpha$ (see Sec. IV B 2).

As a result of an angular increment $\delta \alpha$, all the tangential forces increase because of the increase of the tangential component of the weight which goes like $\sin \alpha$. Consequently, in the distribution, $N \cos \alpha P_{i} \delta \alpha$ sliders shift from the bin $i$ to the bin $i-1$, excepted for the bin No. 1. This process is sketched by the red solid arrows in Fig. 8. For the bin No. 1, $N \cos \alpha P_{1} \delta \alpha$ sliders loose stability. From the qualitative description of the system (Sec. IV B 1), we know that the final value of $\tilde{F}_{t}$ after the rearrangement lies mostly between $\mu_{d} \cos \alpha$ and $\left(\mu_{s}-2 \Delta \mu\right) \cos \alpha$. Thus, after the rearrangments, the sliders remain in bin No. 1 . This process is sketched by the blue dashed arrow in Fig. 8.

This simple process can be put in equations in the form:

$$
\begin{aligned}
\frac{d P_{\nu}}{d \alpha} & =-\frac{1}{2 \Delta \mu} P_{\nu} \\
\frac{d P_{i}}{d \alpha} & =\frac{1}{2 \Delta \mu}\left(P_{i+1}-P_{i}\right) \quad(i \neq 1, \nu) \\
\frac{d P_{1}}{d \alpha} & =\frac{1}{2 \Delta \mu} P_{2}
\end{aligned}
$$

Starting from the condition that the distribution is uni- 
form for $\alpha=0$, thus from $P_{i}(0)=\frac{1}{2 \bar{\mu}_{s}}$, we get:

$$
\begin{aligned}
& P_{\nu}(\alpha)=\frac{1}{2 \bar{\mu}_{s}} e^{-\frac{\alpha}{2 \Delta \mu}} \\
& P_{i}(\alpha)=\frac{1}{2 \bar{\mu}_{s}}\left[\sum_{k=0}^{\nu-i} \frac{1}{k !}\left(\frac{\alpha}{2 \Delta \mu}\right)^{k}\right] e^{-\frac{\alpha}{2 \Delta \mu}} \\
& P_{1}(\alpha)=\frac{1}{2 \bar{\mu}_{s}}\left\{\nu-1-\sum_{k=0}^{\nu-2}\left[\sum_{j=0}^{k} \frac{1}{j !}\left(\frac{\alpha}{2 \Delta \mu}\right)^{j}\right] e^{-\frac{\alpha}{2 \Delta \mu}}\right\}
\end{aligned}
$$

It is of particular interest to focus on the behavior of the solution far from the lower boundary. For realistic values of $\mu_{s}$ and $\mu_{d}, \nu(\alpha)$ remains large and, in the limit, $\nu-i \gg 1$, we have $\sum_{k=0}^{\nu-i} \frac{x^{k}}{k !} \simeq e^{x}(\forall x)$. Consequently, for small angle $\alpha$ so that $\nu(\alpha)$ is still large $\left(\nu(0)=\frac{\bar{\mu}_{s}}{\Delta \mu} \gg 1\right.$ for realistic values of $\mu_{s}$ and $\left.\mu_{d}\right)$ and for $i \ll \nu$, i.e. far from the lower bound of the system, we have:

$$
\begin{aligned}
& P_{i}(\alpha) \simeq \frac{1}{2 \bar{\mu}_{s}} \quad(i \neq 1) \wedge(i \ll \nu) \\
& P_{1}(\alpha) \simeq \frac{1}{2 \bar{\mu}_{s}}\left(1+\frac{\alpha}{2 \Delta \mu}\right)
\end{aligned}
$$

Note that, as is, $P_{1}(\alpha)$ accounts for the amplitude of the peak at large $\tilde{F}_{t}$ as function of the inclination whereas the constant $P_{i}(\alpha)$ correspond to the plateau.

We can now compare our model (Eq. 18) and its limiting case (Eq. 19) to the results of the numerical simulations. To do so, we report in Fig. 9, the probability $P_{i}$ as function of the inclination angle $\alpha$ for the data already reported in Fig. 6.

First, we observe that Eq. (19) correctly describes the linear increase of the amplitude of the peak $P_{1}$ at large $\tilde{F}_{t}$ in a large range of inclination angle $\alpha$ (0 to typically 0.38). Second, Eq. (19) also accounts for the constant value of $P_{i}$ in the same range of inclination, but only for the largest values of $\tilde{F}_{t}$. Slightly less crude, Eq. (18) predicts the decrease of the $P_{i}(i \neq 1)$ upon increasing $\alpha$. However, reporting the probability of the smallest value of $\tilde{F}_{t}$, we observe the model underestimates the depletion of the smallest values of $\tilde{F}_{t}$. This point will be further commented in the discussion (Sec. V).

In spite of this reasonably good agreement between the model and the numerical results, a trend observed in Fig. 9, which is not described at all by the model, is the linear increase of $P_{2}$ starting at $\alpha \simeq 0.2$, followed by a sharp increase for $\alpha \gtrsim 0.4$. We observe that, for $0.2 \lesssim$ $\alpha \lesssim 0.4$, the slope $d P_{2} / d \alpha=\frac{1}{4 \mu_{s} \Delta \mu}$, thus takes the same value that $d P_{1} / d \alpha$. The model fails in accounting for the increase of $P_{2}$ because we did not consider the decrease of the upper bound upon inclination (the maximum force value evolves like $\cos \alpha$ ).

As a matter of fact, due to the shift of the upper bound, the bin No. 2 takes the place of bin No. 1 as the inclination proceeds.

Up-to-now, we commented mainly on the behavior of the system for limited values of the inclination angle

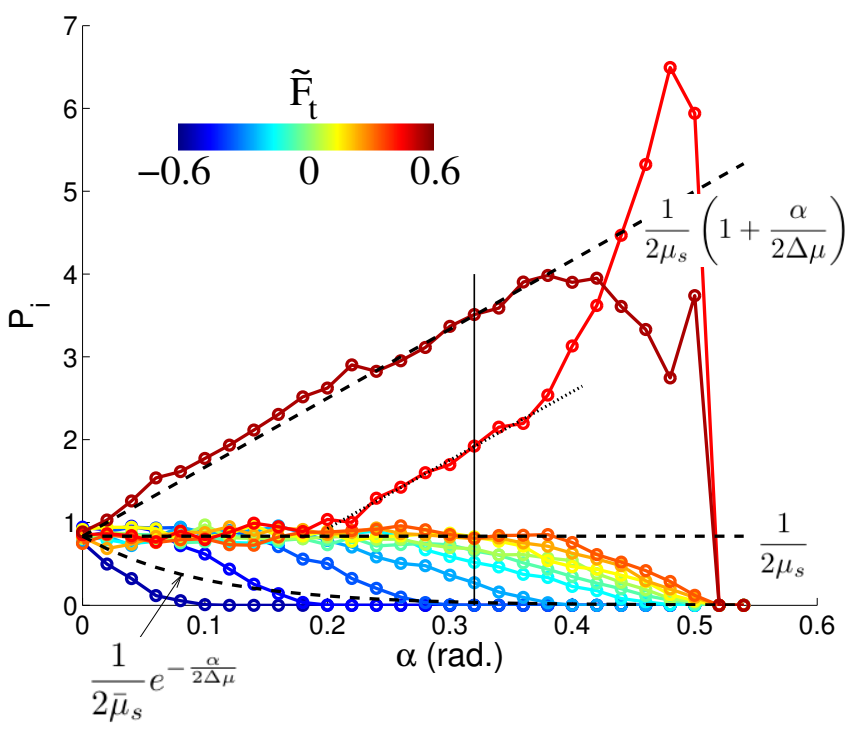

FIG. 9. Height $P_{i}$ vs. angle $\alpha$ for the data of Fig. 6. The dashed straight lines correspond to prediction of the crude model (Eq. 19). The exponential decay of $P_{\nu}(\alpha)$ is given in Eq. (18). The dotted line is a guide for the eye. The vertical solid line is the threshold from Eq. (21) $[N=100, \xi=0$, $\tilde{l}=10, \mu_{d}=0.55, \bar{\mu}_{s}=0.6$, and $\left.\sigma_{\mu}=0.01\right]$.

$\alpha \lesssim 0.38$. For $\alpha \gtrsim 0.38$, a drastic change in the behavior of all the probabilies $P_{i}$ is observed. Indeed, the probability of the largest values, i.e. $P_{1}+P_{2}$, drastically increases whereas the probabilities of smaller values $P_{i}$ $(i>2)$ decreases. One interesting question is whether our model is able to predict the typical angle, $\alpha_{t} \simeq 0.4$, at the transition. To answer the question, consider the probability $P_{3}$ of the force just below the peak:

$$
\begin{aligned}
P_{3}(\alpha) & =\frac{1}{2 \mu_{s}}\left[\sum_{k=0}^{\nu(\alpha)-3} \frac{1}{k !}\left(\frac{\alpha}{2 \Delta \mu}\right)^{k}\right] e^{-\frac{\alpha}{2 \Delta \mu}} \\
& \simeq \frac{1}{2 \mu_{s}}\left(1-\frac{1}{(\nu(\alpha)-2) !}\left(\frac{\alpha}{2 \Delta \mu}\right)^{\nu(\alpha)-2}\right)
\end{aligned}
$$

We previously considered that $\nu(\alpha)$ was large enough for the term on the right-hand-side in the parenthesis to be negligible. Let assume that this is not the case anymore and that this term is of $1 / 10$, i.e. that:

$$
\frac{1}{(\nu(\alpha)-2) !}\left(\frac{\alpha}{2 \Delta \mu}\right)^{\nu(\alpha)-2}=0.1 \text {. }
$$

Using the Stirling approximation and the definition of $\nu(\alpha)$, we get:

$$
\alpha_{t} \simeq \frac{2 \mu_{s}}{1+e} \simeq 0.54 \mu_{s}
$$

which, we point out, does not significantly depend on the choice of the value 1/10 in Eq. (20). We thus get 
$\alpha_{t} \sim 0.32$ for $\bar{\mu}_{s}=0.6$, which underestimates the observed value. However, in regard of the crude approximations we made, we can consider that this last estimate is reasonable.

\section{DISCUSSION}

\section{A. Discussion of the model}

The very simple model of a single slider (Sec. IV A) provides a good understanding of the occurence of the, local, quasi-periodic dynamics since the beginning of the inclination process, i.e. for small tilt angle $\alpha$ (typically $\leq 0.2$ ). Even in a large system, the first sliders that destabilize are isolated from one another and behave like the single slider between two walls, which consist of the two immobile neighbors. The tangential force applied to it increases until it reaches the threshold $\bar{\mu}_{s} \cos \alpha$ [upper dashed line in Fig 2(b)] and then drops by $2\left(\bar{\mu}_{s}-\mu_{d}\right) \cos \alpha$, then reaching the value $\left(2 \mu_{d}-\bar{\mu}_{s}\right) \cos \alpha$ [lower dashed line in Fig 2(b)]. Each rearrangment leads to the increase of the tangential force applied to the neighbors by $\left(\bar{\mu}_{s}-\mu_{d}\right) \cos \alpha$.

Upon inclination, rearrangments involving initially only one slider lead to an increase of the tangential force applied to its neighbors sufficient to destabilize at least one of them. The pattern is then more complex but the picture of a process consisting of a series of, quasiperiodic, localized destabilizations holds. The number of sliders involved in each rearrangment increases upon inclination. When several sliders are involved, the rearrangements repeat with the period $\Delta \alpha \sim\left(\bar{\mu}_{s}-\mu_{d}\right)$ rather than $\Delta \alpha \sim 2\left(\bar{\mu}_{s}-\mu_{d}\right)$ (Fig 2). Indeed, as discussed in Sec. IV B 1, when several sliders enter in motion, the progressive slowing down of the dynamics due to dissipation leads to the value $\mu_{d} \cos \alpha$ [instead of $\left(2 \mu_{d}-\bar{\mu}_{s}\right) \cos \alpha$ for a single slider] of the tangential force.

However, in large systems, in absence of global coupling, the previous picture holds only locally and the overall dynamics is not quasi-periodic (Fig. 3). The rearrangments occuring in different regions are not correlated. The growth in the size of the active regions upon inclination leads them to merge before the whole system looses stability.

The introduction of a global coupling, even small $\left(\xi=10^{-3}\right)$, leads to the synchronisation of the previously isolated local rearrangements and, thus, to the occurence of a quasi-periodic dynamics of the whole system (Fig. 4). This synchronization is reminiscent of the synchronization of oscillators, which is a well-known phenomenon [20].

A particularly interesting, and striking, result is that one can distinguish a clear change in the activity of the system, with or without global coupling, at an inclination angle $\alpha$ of about 0.25 , thus typically half of the avalanche angle $\alpha_{c}$ : for $\alpha \lesssim 0.25$, the system is rather quiescent with few rearrangements occuring locally whereas, for larger $\alpha$, numerous large events of increasing size occur. The model shows that a change in the dynamics is indeed expected for $\alpha_{t} \simeq 0.54 \bar{\mu}_{s}$. For $0 \leq \alpha \lesssim \alpha_{t}$, the number of active sliders increases linearly with $\alpha$. By contrast, for $\alpha \gtrsim \alpha_{t}$, the number of active sliders drastically increases (Fig. 9). In practice, $0.54 \bar{\mu}_{s}$ is close to half the angle of avalanche angle, $\alpha_{c}$, so that we can consider that, to the level of approximation of our model, the transition is well accounted for.

\section{B. Comparison to experiments}

Finally, we underline strong similarities between features of our model and the experiments mentionned in the introduction [2-4].

Upon inclination of a container filled with a granular material, one observed, for small tilt angle, the occurence of small, uncorrelated, rearrangements randomly distributed in the system. At approximately half the avalanche angle, the activity drastically increases in the form of bursts of synchronized displacements.

The order of magnitude of the angular period observed experimentally is typically of a few degrees. In our model, we predict that the periodicity is linked to the difference between the static and the dynamic friction coefficients: $\Delta \alpha \sim\left(\mu_{s}-\mu_{d}\right)$. For granular matter, those friction coefficients can be assimilated to the tangent of, respectively, the angle of avalanche, at which the granular surface looses stability, and of the angle of repose, at which corresponds the granular surface stops flowing. In granular matter $\left(\mu_{s}-\mu_{d}\right) \sim 0.1$ [21] leading thus to $\delta \alpha$ indeed of a few degrees.

In our model, we obtain synchronization only when some global coupling is introduced, which leads to the question of the physical significance of this global coupling in experiments. In the classical Burridge-Knopoff model a rearrangement affects only the immediate neighbors. Such local model is irrealistic for modeling faults as the bulk materials at each side of the fault are of finite stiffness and mediate long-range elastic forces. Taking into account such long-range elastic redistribution of the stress after a reaarrengement has been taken into account in Burridge-Knopoff models by coupling blocks elastically to a various number of distant neighbors [22]. Such long-range elastic coupling has also been discussed in tribology-related studies (see [23] and references therein). In granular matter, it has been shown that when a local, plastic rearrangement takes place, an elastic response with long-ranged redistribution of the stress is observed $[24,25]$. This justifies the introduction of a longrange coupling in the model. Another clue is provided by the observation, in experiments, that the pseudo-period of the bursts depends on the cohesion in the system $[4,5]$. This is in accordance with a picture of a synchronization due to a long-range coupling.

It has to be underlined that our model is not in contradiction with previous works, in particular with studies 
based on cellular automata reported in Ref. [3]. The authors reported an exponential growth of the accumulated plastic activity upon inclination. This trend, also observed experimentally, has been considered as a test validating the model. Such an exponential evolution could seem, at first sight, to be in contradiction with the linear evolution of the number of active sliders which arises in our model. However, the contradiction is only apparent. Actually, reporting the cumulated displacements $A(\alpha)$ in the whole system from the beginning of the inclination process:

$$
A(\alpha)=\sum_{i=0}^{i_{c}} \sum_{n=1}^{N}\left[\tilde{x}_{n}^{(i+1)}-\tilde{x}_{n}^{(i)}\right]
$$

with $\tilde{x}_{n}^{(k)}$ the position of the $n^{t h}$ slider after the $i^{\text {th }}$ rearrangement and $i_{c}$ the total number of rearrangements we also observe a typically exponential increase for our model (Fig. 10a). This is explained by an exponential increase of the typical displacement associated to the rearrangements with the inclination angle $\alpha$ (Fig. 10b).

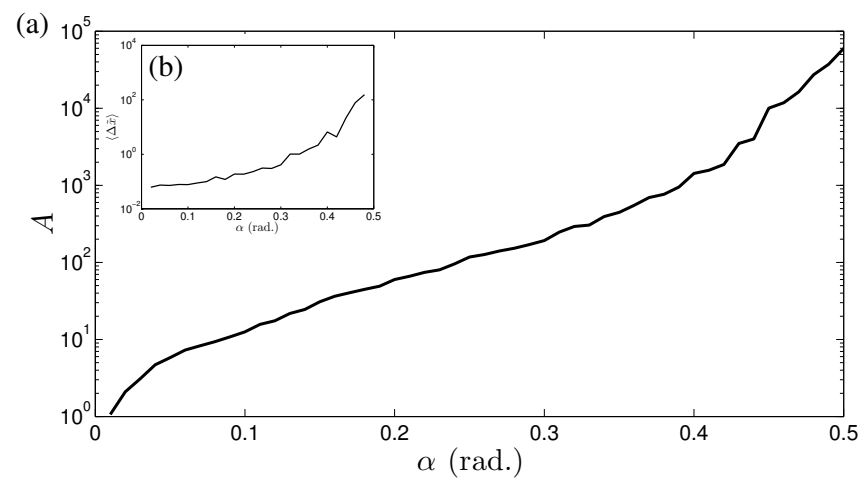

FIG. 10. (a) Cumulated displacement $A$ vs. angle $\alpha$ - Upon inclination, $A$ increases exponentially in spite of the linear increase of the number of sliders involved in the rearrangments with $\alpha$. (b) Average displacement during a rearrangement $\langle\Delta \tilde{x}\rangle$ vs. angle $\alpha . \quad\left[N=100, \xi=0, \tilde{l}=10, \mu_{d}=0.55\right.$, $\bar{\mu}_{s}=0.6$, and $\left.\sigma_{\mu}=0.01\right]$.

\section{CONCLUSION}

We have presented a one-dimensional frictional model consisting of a chain of elastically coupled sliders in frictional contact with an incline. The model reproduces most of the features observed experimentally when quasistatically tilting a box filled with a granular material. In particular, the system reproduces the quasi-periodic series of rearrangments observed in experiments.

We explain the regularity of the bursts of rearrangments as the result of a combination of stick-slip and synchronization due to a large scale coupling. The phenomenon is thus related to the problem of synchronization of oscillators. The coupling originates from the redistribution of the forces, at large scale, in the bulk of the material.

In addition, our toy model provides a statistical approach to describe the evolution of the state of the system which approaches the avalanche. In particular, we revealed an internal threshold before the avalanche occurs. This threshold delimits the transition, at typically half the avalanche angle, from an initial smooth increase of the number of active sliders to an accelerating regime where a dramatic increase of the activity takes place. In regard to the prediction of avalanches, the determination of such a threshold is precious as it delimits a rather regular and predictible evolution of the system and a rapid growth regime which announces the avalanche.

Our model remains simplistic and, in accordance, provides no more than a qualitative description of the phenomenon. In addition, it has to be noted that we considered a unique preparation, consisting of a uniform distribution of the initial tangential forces. Nevertheless, considering that the model is based on very general arguments (disorder, solid friction and coupling), we expect that it unveils the core mechanisms at play in different experimental configurations.
[1] P. Bak, C. Tang, and K. Wiesenfeld, Phys. Rev. Lett. 59, 381 (1987).

[2] N. Nerone, M. A. Aguirre, A. Calvo, D. Bideau, and I. Ippolito, Phys. Rev. E 67, 011302 (2003).

[3] S. Kiesgen de Richter, G. Le Caër, and R. Delannay, J. Stat. Mech.: Theory Exp., (2012) P04013.

[4] A. Amon, R. Bertoni, and J. Crassous, Phys. Rev. E 87, 012204 (2013).

[5] M. Duranteau, V. Tournat, V. Zaitsev, R. Delannay, and P. Richard, AIP Conference Proceedings 1542, 650 (2013).

[6] N. Gravish, and D. I. Goldman, Phys. Rev. E 90, 032202 (2014).

[7] L. Staron, J.-P. Vilotte, and F. Radjai, Phys. Rev. Lett.
89, 204302 (2002).

[8] L. Staron, F. Radjai, and J.-P. Vilotte, J. Stat. Mech. (2006) P07014.

[9] P. Welker and S. McNamara, Granular Matter 13, 93 (2011).

[10] V. B. Nguyen, T. Darnige, A. Bruand, and E. Clement, Phys. Rev. Lett. 107, 138303 (2011).

[11] A. Le Bouil, A. Amon, J.-C. Sangleboeuf, H. Orain, P. Bésuelle, G. Viggiani, P. Chasle, and J. Crassous, Granular Matter, 16, 1 (2014).

[12] S. M. Rubinstein, G. Cohen, and J. Fineberg, Phys. Rev. Lett. 98, 226103 (2007).

[13] R. Burridge and L. Knopoff, Bull. Seismol. Soc. Am. 57, 3411 (1967). 
[14] J. M. Carlson and J.S. Langer, Phys. Rev. Lett. 62, 2632 (1989).

[15] J. M. Carlson, J.S. Langer, and B. E. Shaw, Rev. Mod. Phys. 66, 657 (1994).

[16] O. M. Braun, I. Barel, and M. Urbakh, Phys. Rev. Lett. 103, 194301 (2009).

[17] S. Maegawa, A. Suzuki, and K. Nakano, Tribol. Lett. 38, 313 (2010).

[18] B. Blanc, L. A. Pugnaloni, and J.-C. Géminard, Phys. Rev. E 84, 061303 (2011).

[19] B. Blanc, J.-C. Géminard, and L. A. Pugnaloni, The European Physical Journal E 37, 112 (2014).
[20] S. H. Strogatz and I. Stewart, Scientific American 269, 68 (1993).

[21] H. M. Jaeger, C. H. Liu, and S. R. Nagel, Phys. Rev. Lett. 62, 40 (1989).

[22] J. Xia, H. Gould, W. Klein, and J. B. Rundle, Phys. Rev. Lett. 95, 248501 (2005).

[23] D. S. Amundsen, J. Scheibert, K. Thøgersen, J. Trømborg, and A. Malthe-Sørensen, Tribol. Lett. 45, 357 (2012).

[24] A. Le Bouil, A. Amon, S. McNamara, and J. Crassous, Phys. Rev. Lett. 112, 246001 (2014).

[25] S. McNamara, J. Crassous, and A. Amon, Phys. Rev. E 94, 022907 (2016). 